\title{
THE SYMMETRIC DISCONTINUOUS GALERKIN METHOD DOES NOT NEED STABILIZATION IN 1D FOR POLYNOMIAL ORDERS $p \geq 2$
}

\author{
E. BURMAN ${ }^{1}$, A. ERN, I. MOZOLEVSKI ${ }^{2}$, AND B. STAMM ${ }^{1}$
}

\begin{abstract}
In this Note we prove that in one space dimension, the symmetric discontinuous Galerkin method for second order elliptic problems is stable for polynomial orders $p \geq 2$ without using any stabilization parameter. The method yields optimal convergence rates in both the broken energy norm and the $L^{2}$-norm and can be written in conservative form with fluxes independent of any stabilization parameter. To cite this article: E. Burman, A. Ern, I. Mozolevski and B. Stamm, C. R. Acad. Sci. Paris, vol. 345, num. 10, 2007, p. 599-602.
\end{abstract}

\section{INTRODUCTION}

The Discontinuous Galerkin (DG) method is a classical technique to approximate elliptic and hyperbolic PDE's. A unified theory has been developed recently in the framework of Friedrichs' systems [4]. For elliptic PDE's, two of the most popular methods are the Symmetric Interior Penalty (SIP) method introduced by Baker [2] and Arnold [1] and the Nonsymmetric DG method introduced by Oden, Babuška and Baumann [7]. One attractive feature of the latter method is that, because of the absence of penalty terms, it can be written in conservative form with fluxes that are independent of numerical parameters. Moreover, the Nonsymmetric DG method is proven to yield optimal convergence estimates in the broken energy norm in one [5] and two space dimensions [6] for polynomial orders $p \geq 2$, while for $p=1$, penalty terms must be introduced to grant stability and optimal convergence rates in the broken energy norm, but the conservative fluxes then depend on the penalty parameter.

Working with the SIP method instead of the Nonsymmetric DG method presents the twofold advantage of dealing with symmetric linear systems and of ensuring optimal convergence rates also in the $L^{2}$-norm. The difficulty with the SIP method is that stability usually relies on the use of penalty parameters that will subsequently enter the expression of the conservative fluxes. An exception was provided in the case of polynomial order $p=1$ in [3] where stable SIP methods were proposed in two and three space dimensions without stabilization on interior faces or with stabilization of the diffusive fluxes only.

The purpose of this Note is to fill the gap between Symmetric and Nonsymmetric DG methods in one space dimension. We indeed prove that the symmetric DG method without any penalty leads to optimal convergence rates in the broken energy norm and in the $L^{2}$-norm in one space dimension for polynomial orders $p \geq 2$.

1: Partially supported by the Swiss National Science Foundation under grant 200021-113304.

2: Partially supported by CNPq, Brazil. 
Thus, the present method has only advantages with respect to the Nonsymmetric DG method in the one-dimensional setting. The two-dimensional case for $p \geq 2$ is being currently investigated.

\section{MOdel PROBLEM AND METHOD FORMULATION}

Let $\Omega=(a, b) \subset \mathbb{R}, f \in L^{2}(\Omega)$ and $g_{a}, g_{b} \in \mathbb{R}$. Consider the following boundary value problem:

$$
-u^{\prime \prime}=f \text { in } \Omega, \quad u(a)=g_{a}, \quad u(b)=g_{b} .
$$

This problem is well-posed in $H^{1}(\Omega)$ and since $f \in L^{2}(\Omega)$, its unique solution is in $H^{2}(\Omega)$. Let $\mathcal{K}_{h}$ be a partition of the domain $\Omega$ formed by $M$ elements $K_{i}=$ $\left(x_{i}, x_{i+1}\right)$. For simplicity, $\mathcal{K}_{h}$ is assumed to be uniform, i.e., $x_{i}=a+i h, i \in$ $\{0, \ldots, M\}$ where $h=\frac{b-a}{M}$ denotes the mesh size. Let an integer $p \geq 0$ and consider the usual discontinuous finite element space

$$
V_{h}^{p}=\left\{v \in L^{2}(\Omega) ; \forall i \in\{1, \ldots, M\},\left.v\right|_{K_{i}} \in \mathbb{P}_{p}\left(K_{i}\right)\right\},
$$

where $\mathbb{P}_{p}\left(K_{i}\right)$ denotes the $p$-th order polynomial space on $K_{i}$. Let $\mathcal{N}_{h}$ denote the set of all nodes of $\mathcal{K}_{h}$ and let $\mathcal{N}_{h}^{\mathrm{i}}$ denote the set of all interior nodes. For any function $v \in H^{1}\left(\mathcal{K}_{h}\right)$, where for any $s \geq 1, H^{s}\left(\mathcal{K}_{h}\right)$ denotes the usual broken Sobolev space of order $s$, define its jump and average at interior nodes as follows:

$$
[[v]]_{i}=\left.v\right|_{K_{i}}\left(x_{i}\right)-\left.v\right|_{K_{i+1}}\left(x_{i}\right), \quad\{v\}_{i}=\frac{1}{2}\left(\left.v\right|_{K_{i+1}}\left(x_{i}\right)+\left.v\right|_{K_{i}}\left(x_{i}\right)\right) .
$$

On boundary nodes, the following notation is used: $\left[[v]_{0}=-v(a),[[v]]_{M}=v(b)\right.$, $\{v\}_{0}=v(a)$ and $\{v\}_{M}=v(b)$. For any region $R \subset \Omega$ composed of one or more mesh cells, $(\cdot, \cdot)_{R}$ denotes the usual $L^{2}(R)$-scalar product and $\|\cdot\|_{R}$ the associated norm. In the sequel, the inequality $A \lesssim B$ means that there is a positive $c$, independent of $h$, such that $A \leq c B$. For simplicity, the dependency of the constants on $p$ is not addressed herein.

The symmetric DG method consists of finding $u_{h} \in V_{h}^{p}$ such that

$$
a_{h}\left(u_{h}, v_{h}\right)=l\left(v_{h}\right), \forall v_{h} \in V_{h}^{p}
$$

where

$$
\begin{aligned}
a_{h}\left(u_{h}, v_{h}\right) & =\sum_{K_{i} \in \mathcal{K}_{h}}\left(u_{h}^{\prime}, v_{h}^{\prime}\right)_{K_{i}}-\sum_{x_{i} \in \mathcal{N}_{h}}\left(\left[\left[u_{h}\right]\right]_{i}\left\{v_{h}^{\prime}\right\}_{i}+\left\{u_{h}^{\prime}\right\}_{i}\left[\left[v_{h}\right]\right]_{i}\right) \\
l\left(v_{h}\right) & =\sum_{K_{i} \in \mathcal{K}_{h}}\left(f, v_{h}\right)_{K_{i}}+g_{a} v_{h}^{\prime}(a)-g_{b} v_{h}^{\prime}(b) .
\end{aligned}
$$

Observe that the jumps of the discrete solution at interior nodes are not penalized and that the boundary conditions are not enforced by penalty but just through the consistency terms, i.e., the contribution of boundary nodes in the last term of Eq. (4). Furthermore, the discrete problem (3) is consistent. Indeed, integration by parts yields for any $v, w \in H^{2}\left(\mathcal{K}_{h}\right)$,

$$
a_{h}(v, w)=-\sum_{K_{i} \in \mathcal{K}_{h}}\left(v^{\prime \prime}, w\right)_{K_{i}}-\sum_{x_{i} \in \mathcal{N}_{h}}[[v]]_{i}\left\{w^{\prime}\right\}_{i}+\sum_{x_{i} \in \mathcal{N}_{h}^{\mathrm{i}}}\left[\left[v^{\prime}\right]\right]_{i}\{w\}_{i} .
$$

Applying this with $v:=u$, the solution to (1), and $w:=v_{h}$ arbitrary in $V_{h}^{p}$ yields $a_{h}\left(u, v_{h}\right)=l\left(v_{h}\right)$. 


\section{Convergence AnAlysis}

Define the following triple norm in $H^{1}\left(\mathcal{K}_{h}\right)$ :

$$
\|v\|\left\|^{2}=\right\| v^{\prime} \|_{\mathcal{K}_{h}}^{2}+\sum_{x_{i} \in \mathcal{N}_{h}} \frac{1}{h}\left[[v]_{i}^{2} \quad \text { where } \quad\left\|v^{\prime}\right\|_{\mathcal{K}_{h}}^{2}=\sum_{K_{i} \in \mathcal{K}_{h}}\left\|v^{\prime}\right\|_{K_{i}}^{2} .\right.
$$

The main technical result of this section is the following

Lemma 3.1. Assume $p \geq 2$. Then,

$$
\forall v_{h} \in V_{h}^{p}, \quad\left\|v_{h}\right\| \mid \lesssim \sup _{0 \neq w_{h} \in V_{h}^{p}} \frac{a_{h}\left(v_{h}, w_{h}\right)}{\left\|w_{h}\right\| \mid}
$$

Proof. Let $v_{h} \in V_{h}^{p}$.

(i) Let us prove that there is (a unique) $y_{h} \in V_{h}^{p}$ such that

$$
\left\{\begin{aligned}
\left(y_{h}, z_{h}\right)_{\Omega} & =0, & & \forall z_{h} \in V_{h}^{p-2}, \\
\left\{y_{h}^{\prime}\right\}_{i} & =\frac{1}{h}\left[v_{h}\right]_{i}, & & \forall i \in\{0, \ldots, M\}, \\
\left\{y_{h}\right\}_{i} & =0, & & \forall i \in\{1, \ldots, M-1\} .
\end{aligned}\right.
$$

To this purpose, let us first establish the a priori estimate

$$
\left\|y_{h}\right\|\left|\lesssim\left\|v_{h}\right\|\right|
$$

Since $y_{h} \perp V_{h}^{p-2}$ and $p \geq 2, y_{h}$ has zero mean over each mesh cell. As a result, $y_{h}$ satisfies for all $i \in\{1, \ldots, M\}$, the strong Poincaré inequality

$$
\left\|y_{h}\right\|_{K_{i}} \lesssim h\left\|y_{h}^{\prime}\right\|_{K_{i}} .
$$

Hence, using a trace inequality yields

$$
\sum_{x_{i} \in \mathcal{N}_{h}} \frac{1}{h}\left[\left[y_{h}\right]\right]_{i}^{2} \lesssim \frac{1}{h^{2}} \sum_{K_{i} \in \mathcal{K}_{h}}\left\|y_{h}\right\|_{K_{i}}^{2} \lesssim\left\|y_{h}^{\prime}\right\|_{\mathcal{K}_{h}}^{2} .
$$

Moreover, integrating by parts and using the properties of $y_{h}$, it is inferred that

$$
\begin{aligned}
\left\|y_{h}^{\prime}\right\|_{\mathcal{K}_{h}}^{2} & =-\sum_{K_{i} \in \mathcal{K}_{h}}\left(y_{h}^{\prime \prime}, y_{h}\right)_{K_{i}}+\sum_{x_{i} \in \mathcal{N}_{h}^{i}}\left[\left[y_{h}^{\prime}\right]\right]_{i}\left\{y_{h}\right\}_{i}+\sum_{x_{i} \in \mathcal{N}_{h}}\left[\left[y_{h}\right]_{i}\left\{y_{h}^{\prime}\right\}_{i}\right. \\
& =\sum_{x_{i} \in \mathcal{N}_{h}}\left[[ y _ { h } ] _ { i } \frac { 1 } { h } \left[\left[v_{h}\right]_{i} \lesssim\left\|v_{h}\right\| \|\left(\sum_{x_{i} \in \mathcal{N}_{h}} \frac{1}{h}\left[\left[y_{h}\right]_{i}^{2}\right)^{\frac{1}{2}} \lesssim\left\|v_{h}\right\|\left\|y_{h}^{\prime}\right\|_{\mathcal{K}_{h}},\right.\right.\right.
\end{aligned}
$$

whence the a priori estimate (9) readily follows. To conclude this first step of the proof, it now suffices to observe that (8) is nothing more than a square linear system of size $(p+1) M$. Hence, the existence of $y_{h}$ is equivalent to the fact that the matrix associated with (8) has zero kernel, which, in turn, is a straightforward consequence of the a priori estimate (9).

(ii) Owing to (6) and (8),

$$
a_{h}\left(v_{h},-y_{h}\right)=\sum_{x_{i} \in \mathcal{N}_{h}} \frac{1}{h}\left[\left[v_{h}\right]_{i}^{2} .\right.
$$

Furthermore, using a trace inequality leads to

$$
a_{h}\left(v_{h}, v_{h}\right)=\left\|v_{h}^{\prime}\right\|_{\mathcal{K}_{h}}^{2}-2 \sum_{x_{i} \in \mathcal{N}_{h}}\left[\left[v_{h}\right]_{i}\left\{v_{h}^{\prime}\right\}_{i} \geq \frac{1}{2}\left\|v_{h}^{\prime}\right\|_{\mathcal{K}_{h}}^{2}-c \sum_{x_{i} \in \mathcal{N}_{h}} \frac{1}{h}\left[\left[v_{h}\right]_{i}^{2},\right.\right.
$$





FiguRE 1. $L^{2}(\Omega)$ (left) and $H^{1}\left(\mathcal{K}_{h}\right)$ (right) norm of the error as function of the mesh diameter $h$.

with $c$ independent of $h$. Hence, there is $\lambda$ large enough such that $\left.\left\|v_{h}\right\|\right|^{2} \lesssim$ $a_{h}\left(v_{h}, v_{h}-\lambda y_{h}\right)$, whence $(7)$ is readily inferred owing to (9).

Remark 1. The proof of Lemma 3.1 breaks down for $p=1$ because in this case, it can not be inferred that $y_{h}$ has zero mean elementwise and thus the strong Poincaré inequality cannot be used. A direct analysis for the case $p=1$ shows that the matrix associated with the bilinear form $a_{h}$ on $V_{h}^{1}$ is singular with one-dimensional kernel spanned by the so-called checkerboard mode (the function in $V_{h}^{0}$ equal to \pm 1 on alternating mesh cells). This matrix becomes symmetric definite positive if the bilinear form $a_{h}$ is supplemented by penalizing a jump at an interior node or one boundary value. In the multidimensional case with $p=1$ the checkerboard mode can be controlled by the mesh geometry (see [3]).

Owing to (7) and using standard finite element techniques for error estimates leads to the main convergence result of this Note.

Theorem 3.2. Let $u \in H^{r}\left(\mathcal{K}_{h}\right) \bigcap H^{2}(\Omega), r \geq 2$, solve (1) and let $u_{h} \in V_{h}^{p}, p \geq 2$, solve (3). Then, for all $1 \leq s \leq \min (p+1, r)$,

$$
\left\|u-u_{h}\right\|_{\Omega}+h\|\| u-u_{h}\left\|\leq c h^{s}\right\| u \|_{H^{s}\left(\mathcal{K}_{h}\right)}
$$

\section{Numerical test}

To illustrate the convergence result of Theorem 3.2, consider $\Omega=(0,1)$ with homogeneous boundary conditions and with $f$ such that the solution is $u(x)=$ $\sin (12 \pi x) e^{1.75 x}$. Figure 1 presents the convergence rates in the $H^{1}\left(\mathcal{K}_{h}\right)$ - and $L^{2}(\Omega)$ norms for a sequence of nested uniform meshes and for approximation orders $p \in$ $\{2, \ldots, 7\}$. Remark on the dependency on $p$ of the inf-sup constant once Igor gets the results for that.. 


\section{REFERENCES}

[1] D. N. Arnold. An interior penalty finite element method with discontinuous elements. SIAM J. Numer. Anal., 19(4):742-760, 1982.

[2] G. Baker. Finite element methods for elliptic equations using nonconforming elements. Math. Comp., 31:44-59, 1977.

[3] E. Burman and B. Stamm. Low order discontinuous Galerkin methods for second order elliptic problems. Technical Report 04-2007, EPFL-IACS, 2007.

[4] A. Ern and J.-L. Guermond. Discontinuous Galerkin methods for Friedrichs' systems. I. General theory. SIAM J. Numer. Anal., 44(2):753-778, 2006.

[5] M. G. Larson and A. J. Niklasson. Analysis of a family of discontinuous Galerkin methods for elliptic problems: the one dimensional case. Numer. Math., 99:113-130, 2004.

[6] M. G. Larson and A. J. Niklasson. Analysis of a nonsymmetric discontinuous Galerkin method for elliptic problems: stability and energy error estimates. SIAM J. Numer. Anal., 42(1):252264,2004

[7] J. T. Oden, I. Babuška, and C. Baumann. A discontinuous $h p$ finite element method for diffusion problems. J. Comput. Phys., 146(2):491-519, 1998.

E. Burman, iacs/CMCS, Station 8, Ecole Polytechnique Fédérale de Lausanne, CH 1015, Lausanne, Switzerland.

E-mail address: Erik.Burman@epfl.ch

A. Ern, Cermics, Ecole des Ponts, Paristech, 6 \& 8 Av. B. Pascal, 77455 MarneLa-Vallée Cedex 2, France.

E-mail address: ern@cermics.enpc.fr

I. Mozolevski, Mathematics Department, Federal University of Santa Catarina, SC, 88040-900, Florianópolis, Brazil and Cermics, Ecole des Ponts, ParisTech, 6 \& 8 Av. B. Pascal, 77455 Marne-la-Vallée cedex 2, France.

E-mail address: Igor.Mozolevski@mtm.ufsc.br

B. Stamm, iacs/CmCS, Station 8, Ecole Polytechnique Fédérale de Lausanne, CH 1015,Lausanne, Switzerland.

E-mail address: Benjamin.Stamm@epfl.ch 